\title{
Factores asociados a la efectividad de safenectomía total o parcial en pacientes intervenidos en el Hospital Militar Central, Lima, Perú
}

\author{
Associated factors for the effectiveness of total \\ or partial saphenectomy in patients treated at the \\ Peruvian Central Military Hospital, Lima, Peru
}

Correspondencia Grethel Lorena Serván Chávez loreka_09@hotmail.com

\section{Recibido: 30/04/2018}

Arbitrado por pares

Aprobado: 18/07/2018

Citar como: Serván-Chávez $G$, Sánchez-Bardales F, Chávez-Peche J, Angulo-Bazán Y. Factores asociados a la efectividad de safenectomía total o parcial en pacientes intervenidos en el Hospital Militar Central, Lima, Perú. Acta Med Peru. 2018;35(3):153-9
Grethel Serván-Chávez ${ }^{1}$, Fernando Sánchez-Bardales ${ }^{1,2}$, José Chávez-Peche ${ }^{3}$, Yolanda Angulo-Bazán ${ }^{4,5}$

1 Escuela de Medicina Humana, Universidad Privada Antenor Orrego. Trujillo, Perú.

2 Hospital de Alta Complejidad Virgen de la Puerta. Trujillo, Perú.

3 Hospital Militar Central Coronel Luis Arias Schreiber. Lima, Perú.

4 CIGES-UFRO. Universidad de la Frontera. Temuco, Chile.

5 Gerencia Central de Prestaciones de Salud, Seguro Social de Salud. Lima, Perú.

\section{RESUMEN}

Objetivo: Evaluar factores asociados a la efectividad de la safenectomía parcial (SP) y total (ST) en pacientes intervenidos en el Hospital Militar Central (HMC) "Luis Arias Schreiber" durante el año 2017. Materiales y métodos: Estudio transversal. Se evaluaron historias clínicas de pacientes operados por insuficiencia venosa periférica en el HMC durante el periodo de enero - diciembre del año 2017. Se evaluaron variables como edad, género, comorbilidades (diabetes tipo 2, hipertensión arterial y obesidad), tiempo de enfermedad (en años), ubicación quirúrgica, tiempo operatorio, presencia de complicaciones (hematoma, infección de sitio operatorio y lesión del nervio safeno), días de hospitalización y mejoría clínica referida. Se buscó asociación entre estas variables y el tipo de safenectomía realizada (SP o ST) mediante la prueba de Chi cuadrado, prueba exacta de Fisher, razones de prevalencias (RP) y U de MannWhitney. Adicionalmente se evaluaron factores asociados a la lesión del nervio safeno en el grupo de ST, mediante regresión logística. Resultados: Se evaluaron 107 historias clínicas, 43 personas se sometieron a SP $(40,2 \%)$ y 64 a ST $(59.8 \%)$, con una mediana de edad de 58 años, en su mayor parte varones $(64,5 \%)$. No hubo diferencias significativas entre las características clínico-epidemiológicas prequirúrgicas y el tipo de cirugía a la que se sometieron. Se encontró una menor proporción de complicaciones posoperatorias [RP (IC95\%): 0,06 $(0,01-0,22)]$, lesión del nervio safeno [RP (IC95\%): 0,02 (0,00-0,19)] y días de hospitalización $(p<0,05)$ en los pacientes con SP. Conclusión: pacientes con SP presentaron mejores desenlaces posoperatorios (frecuencia de complicaciones posoperatorias, lesión del nervio safeno y días de hospitalización) en comparación con los que recibieron ST. Futuros estudios deberían estudiar las posibles variables intervinientes en estos desenlaces.

Palabras clave: Insuficiencia venosa; Complicaciones posoperatorias; Venas varicosas; Procedimientos quirurgicos vasculares (fuente: DeCS BIREME). 


\section{ABSTRACT}

Objective: To assess associated factors for the effectiveness of partial (PS) and total (TS) saphenectomy performed in patients from the Luis Arias-Schereiber Central Military Hospital (CMH) during 2017. Materials and methods: This is a cross-sectional study. Clinical records from $\mathrm{CMH}$ patients who underwent surgery because of peripheral venous insufficiency from January to December 2017 were reviewed. The following variables were assessed: age, gender, comorbidities (type 2 diabetes, high blood pressure, and obesity), time with the disease (in years), surgical location, operative time, occurrence of complications (hematoma, operative site infection, and saphenous nerve lesion), days of hospital stay, and declared clinical improvement. Associations amongst these variables and the type of performed saphenectomy (PS or TS) were sought for by using the Chi-square test, Fisher's exact test, prevalence rates (PR), and the Mann-Whitney U test. Also, factor associated to a lesion in the saphenous nerve were assessed in the TS group using logistic regression. Results: One-hundred and seven clinical records were reviewed, 43 persons underwent PS (40.2\%) and 64 underwent TS (59.8\%), their mean age was 58 years, and nearly two-thirds of all patients were male (64.5\%). There were no significant differences with respect to presurgical clinical and epidemiological characteristics and the type of surgical procedure performed. There were low rates of postoperative complications [PR $(95 \% \mathrm{Cl}): 0.06(0.01-0.22)$ ], saphenous nerve lesions [PR ( $95 \% \mathrm{Cl}): 0.02(0.00-0.19)]$, and days hospitalized $(p<0.05)$ in patients who underwent PS. Conclusion: Patients who underwent PS had better postoperative outcomes (frequency of postoperative complications, saphenous nerve lesions and hospitalization days) compared to those who had undergone TS. Future studies should assess the variables that might lead to these outcomes.

Keywords: Venous insufficiency; Postoperative complications; Varicose veins; vascular surgical procedures (source: MeSH NLM).

\section{INTRODUCCIÓN}

La insuficiencia venosa crónica (IVC) se define como la condición de incompetencia del sistema valvular de las extremidades inferiores causando un estado persistente y crónico de hipertensión venosa ${ }^{[1,2]}$. Esta patología se manifiesta típicamente mediante síntomas y signos como dolor, pesadez, calambres, edema, cambios en la piel y ulceración ${ }^{[3]}$; todo ello lleva a la aparición de vasos dilatados, palpables y con trayecto tortuoso -usualmente con un diámetro mayor de $3 \mathrm{~mm}$-, pertenecientes a las venas safenas, tributarias y/o superficiales ${ }^{[4]}$.

El tratamiento de primera línea de la IVC tiene como objetivo principal aliviar de signos y síntomas del paciente, prevenir las complicaciones y evitar la progresión de la misma mediante medidas conservadoras (compresión, escleroterapia, ablación intravenosa por medio de láser, agua o radiofrecuencia, entre otros) asociadas a ejercicio físico y alimentación saludable ${ }^{[5,6]}$.

Sin embargo, en el caso de pacientes con marcada sintomatología, con discapacidad, varices prominentes o con ulceras no cicatrizantes, el tratamiento quirúrgico está indicado ${ }^{[7]}$. La cirugía consiste en la ablación de la vena safena incompetente y presenta distintas variantes como la extracción proximal con inversión del vaso, extracción distal con o sin invaginación, safenectomía parcial y safenectomía total ${ }^{[8]}$. De ellas, las dos últimas técnicas son las más frecuentemente usadas en nuestro entorno ${ }^{[9]}$, definiéndose la safenectomia total como la extracción de la vena safena mayor desde la región inguinal hasta el tobillo; mientras que en la safenectomía parcial se extrae la vena safena mayor desde la región inguinal hasta $4 \mathrm{~cm}$ por debajo de la articulación de la rodilla ${ }^{[10]}$.

Si bien la safenectomía en sus diversas modalidades ha mostrado bajas tasas de recurrencia en estudios previos ${ }^{[8,11]}$; presenta también un mayor riesgo de aparición de complicaciones como hematomas, infección de herida operatoria y la lesión del nervio safeno; siendo esta complicación una de las más severas y la que más se busca evitar en las diferentes técnicas antes descritas ${ }^{[10,12]}$.

En nuestro país, no se han encontrado muchos reportes previos sobre el impacto de esta enfermedad en la población peruana, pero se estima que representa aproximadamente el $8 \%$ de enfermedades ocupacionales ${ }^{[13]}$, en donde uno de los grupos más afectados son el personal de las Fuerzas Armadas ${ }^{[14]}$. A pesar de ello, la Guía de Práctica Clínica para Diagnóstico y Tratamiento de la Insuficiencia Venosa Crónica del Hospital Militar Central insiste en recomendar el uso de ablación total de la vena safena mayor sin considerar el riesgo latente de lesión del nervio safeno ${ }^{[15]}$. Parcialmente, esto puede deberse a la escasa información científica local disponible sobre el tema, lo que no permite conocer la realidad de este problema en nuestro país y con ello mejorar las estrategias terapéuticas.

Por ello, el objetivo del presente estudio es evaluar factores asociados a la efectividad de la safenectomía parcial y total en pacientes intervenidos en el Hospital Militar Central (HMC) "Luis Arias Schreiber" durante el año 2017.

\section{MATERIALES Y MÉTODOS}

\section{Diseño y población de estudio}

Estudio transversal. Se evaluaron todas las historias clínicas de pacientes que fueron sometidos a safenectomía parcial o total por insuficiencia venosa periférica en el Servicio de Cirugía de Tórax y Cardiovascular del HMC "Coronel Luis Arias Schreiber" desde el 1 de enero hasta el 31 de diciembre del 2017. Se incluyeron solo historias clínicas de pacientes que acudieron 
a todos sus controles posoperatorios y se excluyeron aquellos registros de pacientes con patología articular neurológica o de alguna otra índole que provoque dolor de miembros inferiores; $y$ de quienes fueron sometidos a cirugías distintas a safenectomía, o requirieron manejo hospitalario por otra especialidad.

\section{Variables}

Se evaluaron variables demográficas como edad, género y comorbilidades (diabetes tipo 2, hipertensión arterial y obesidad); variables clínicas: tiempo de enfermedad (en años), ubicación quirúrgica, tiempo operatorio e indicadores de desenlace posoperatorio: presencia de complicaciones (hematoma, infección de sitio operatorio y lesión del nervio safeno), días de hospitalización posoperatoria y mejoría clínica (definida como el registro en la historia clínica de la disminución de dolor, sensación de pesadez y/o aparición de calambres, durante el tiempo de hospitalización). Todas las variables fueron agrupadas por el tipo de safenectomía realizada (parcial o total).

\section{Procedimientos o intervenciones}

La investigación fue conducida del 4 de febrero al 16 de marzo del 2018. Se solicitó la autorización correspondiente a la dirección médica del Hospital Militar Central; luego del cual se acudió al Departamento de Cirugía de Tórax y Cardiovascular para identificar el número de las historias clínicas de interés que se obtuvieron por medio del libro de registro de procedimientos quirúrgicos durante el periodo comprendido entre enero a diciembre del 2017. Con el registro obtenido se acudió al archivo de historias clínicas donde se recolectaron los datos sobre las variables antes mencionadas.

\section{Aspectos éticos}

El estudio contó con el permiso del Comité de Investigación y Ética de la Universidad Privada Antenor Orrego (UPAO). En todo momento se preservó el anonimato de las historias clínicas incluidas en el estudio, así como no se registró dato alguno que permitiera la identificación de algún paciente. Como la fuente de datos fue la historia clínica, este estudio no representó riesgo alguno para los pacientes, cuyos registros fueron incluidos.

\section{Análisis de datos}

Se calcularon medianas y rangos intercuartilares en las variables cuantitativas que no cumplían con los criterios de normalidad, así como frecuencias absolutas y relativas para las variables cualitativas. Para establecer diferencias significativas entre las variables de estudio y el tipo de safenectomía, se usó la $U$ de Mann-Whitney (variables cuantitativas); y la prueba Exacta de Fisher o Chi Cuadrado (variables cualitativas) calculándose razones de prevalencias (RP) con sus respectivos intervalos de confianza (IC95\%), asumiendo el uso de safenectomías parciales como exposición. Adicionalmente, se realizó un análisis multivariado de regresión logística para identificar posibles factores asociados a lesión del nervio safeno en el grupo de safenectomías totales, debido a que es una de las complicaciones más frecuentes y severas en esta técnica ${ }^{[16]}$. Se consideró estadísticamente significativo un $p<0,05$, con un nivel de confianza del $95 \%$. Todas las pruebas estadísticas fueron calculadas con apoyo del paquete estadístico IBM SPSS Statistics for Windows, Version 25.0. Armonk, NY: IBM Corp. 2017. ${ }^{\circledR}$.

\section{RESULTADOS}

Se incluyeron en el análisis 107 historias clínicas de 38 mujeres $(35,5 \%)$ y 69 varones $(64,5 \%)$, con una mediana de edad de 58 años. De estas, 43 correspondieron a safenectomías parciales $(40,2 \%)$ y 64 a safenectomías totales $(59,8 \%)$. Al analizar las principales características clínicas y demográficas no se encontraron diferencias significativas entre los grupos. (Tabla 1).

Al evaluar los indicadores posoperatorios por tipo de técnica quirúrgica se encontraron diferencias significativas entre la presencia de complicaciones posoperatorias con una [RP (IC95\%): $0,06(0,01-0,22) ; p<0,001]$ y la cantidad de días de hospitalización $(p=0,001)$, los cuales fueron mayores en el grupo que se realizó safenectomías totales.

Así mismo, se encontró que el $48,4 \%$ de pacientes que se operaron mediante la técnica de safenectomia total resultaron con lesión del nervio safeno encontrándose diferencias significativas al comparar esta situación con el grupo de pacientes que recibió safenectomías parciales [RP (IC95\%): 0,02 (0,00-0,19); $p<0,001]$. No se encontraron diferencias significativas en cuanto a mejoría clínica referida por tipo de técnica quirúrgica (Tabla 2).

En los pacientes que fueron sometidos a una safenectomía parcial, no se encontraron casos de infecciones de sitio operatorio; y solo se encontró un caso de lesión del nervio safeno en una paciente de sexo femenino, de 67 años de edad, con antecedentes de hipertensión arterial y diabetes tipo 2; cuya intervención quirúrgica fue realizada en el lado derecho y a la que se le agregó la aparición de un hematoma como complicación adicional.

De forma similar, en el grupo de safenectomías totales, solo se encontró un caso de infección de sitio operatorio en una paciente de sexo femenino, de 37 años de edad, sin antecedentes patológicos; cuya intervención quirúrgica fue realizada en el lado derecho.

Al evaluar el efecto de posibles factores asociados a la lesión de nervio safeno en el grupo de safenectomias totales; se encontró que el tiempo operatorio menor de 60 minutos puede ser un factor protector para evitar esta complicación [RP (IC95\%): 0,26 $(0,08-0,79) ; p=0,018]$. (Tabla 3).

\section{DISCUSIÓN}

Al describir las variables demográficas, se encontró una mayor proporción de personas del sexo masculino $(64,5 \%)$, con una mediana de 58 años. Es conocido que la frecuencia de insuficiencia venosa va aumentando en forma directamente 
Tabla 1. Distribución de pacientes según características clínicas y técnica quirúrgica. Hospital Militar Central (HMC), 2017.

\begin{tabular}{|c|c|c|c|}
\hline \multirow{2}{*}{ Características clínicas } & Safenectomía parcial & Safenectomía total & \multirow{2}{*}{ Valor $\boldsymbol{p}^{*}$} \\
\hline & $(n=43)$ & $(n=64)$ & \\
\hline Edad (años) & $57(23)$ & $59(22,5)$ & 0,669 \\
\hline \multicolumn{4}{|l|}{ Grupo etario } \\
\hline$<55$ años & $20(18,7 \%)$ & $24(22,4 \%)$ & \multirow{2}{*}{0,353} \\
\hline$\geq 55$ años & $23(21,5 \%)$ & $40(37,4 \%)$ & \\
\hline \multicolumn{4}{|l|}{ Sexo } \\
\hline Femenino & $14(13,1 \%)$ & $24(22,4 \%)$ & \multirow{2}{*}{0,6} \\
\hline Masculino & $29(27,1 \%)$ & $40(37,4 \%)$ & \\
\hline \multicolumn{4}{|l|}{ Comorbilidades } \\
\hline Diabetes tipo 2 & $4(3,7 \%)$ & $4(3,7 \%)$ & 0,711 \\
\hline Hipertensión arterial & $7(6,5 \%)$ & $12(11,2 \%)$ & 0,743 \\
\hline Obesidad & $11(10,3 \%)$ & $7(6,5 \%)$ & 0,083 \\
\hline Tiempo de enfermedad (años) & $4(6)$ & $4(6)$ & 0,921 \\
\hline$\leq 4$ años & $22(20,6 \%)$ & $33(30,8 \%)$ & \multirow{2}{*}{0,968} \\
\hline$>4$ años & $21(19,6 \%)$ & $31(29,0 \%)$ & \\
\hline \multicolumn{4}{|l|}{ Ubicación quirúrgica } \\
\hline Derecha & $15(14,0 \%)$ & $27(25,2 \%)$ & \multirow{3}{*}{0,706} \\
\hline Izquierda & $17(15,9 \%)$ & $24(22,4 \%)$ & \\
\hline Bilateral & $11(10,3 \%)$ & $13(12,1 \%)$ & \\
\hline Tiempo operatorio (minutos) & $60(50)$ & $70(30)$ & 0,465 \\
\hline$<70 \min$ & $23(21,5 \%)$ & $30(28,0 \%)$ & \multirow{2}{*}{0,502} \\
\hline$\geq 70 \mathrm{~min}$ & $20(18.7 \%)$ & $34(31.8 \%)$ & \\
\hline
\end{tabular}

Variables cuantitativas expresadas en medianas (rangos intercuartilares).

* U de Mann-Whitney en el caso de variables cuantitativas y Prueba de Chi cuadrado en variables cualitativas.

Tabla 2. Dimensiones de efectividad post-operatoria por tipo de técnica quirúrgica. Hospital Militar Central (HMC), 2017.

\begin{tabular}{|c|c|c|c|c|}
\hline \multirow{2}{*}{ Efectividad posoperatoria } & Safenectomía parcial & Safenectomía total & $\mathbf{R P}$ & \multirow{2}{*}{ Valor $p$} \\
\hline & $(n=43)$ & $(n=64)$ & (IC95\%) & \\
\hline \multicolumn{5}{|l|}{ Complicaciones posoperatorias } \\
\hline Sí & $3(2,8 \%)$ & $35(32,7 \%)$ & \multirow{2}{*}{$0,06(0,01-0,22)$} & \multirow{2}{*}{$<0,001$} \\
\hline No & $40(37,4 \%)$ & $29(27,1 \%)$ & & \\
\hline \multicolumn{5}{|l|}{ Hematoma } \\
\hline Sí & $3(6,9)$ & $7(10,9)$ & \multirow{2}{*}{$0,61(0,15-2,50)$} & \multirow{2}{*}{0,737} \\
\hline No & $40(93,1)$ & $57(89,1)$ & & \\
\hline \multicolumn{5}{|l|}{ Infección de sitio operatorio } \\
\hline Sí & $0(0,0 \%)$ & $1(0,9 \%)$ & \multirow{2}{*}{ NA } & \multirow{2}{*}{ NA } \\
\hline No & $43(42,0 \%)$ & $63(58,9 \%)$ & & \\
\hline \multicolumn{5}{|l|}{ Lesión de nervio safeno } \\
\hline Sí & $1(0,9 \%)$ & $31(29,0 \%)$ & \multirow{2}{*}{$0,02(0,00-0,19)$} & \multirow{2}{*}{$<0,001$} \\
\hline No & $42(39,3 \%)$ & $33(30,8 \%)$ & & \\
\hline $\begin{array}{l}\text { Días de hospitalización } \\
\text { postoperatoria }\end{array}$ & $1(0)$ & $2(1)$ & & $0,001^{(*)}$ \\
\hline \multicolumn{5}{|l|}{ Mejoría clínica referida } \\
\hline Sí & $41(38,3 \%)$ & $59(55,1 \%)$ & \multirow{2}{*}{$1,74(0,32-9,39)$} & \multirow{2}{*}{0,699} \\
\hline No & $2(1.9 \%)$ & $5(4,7 \%)$ & & \\
\hline
\end{tabular}

RP: razón de prevalencias, tomando el uso de safenectomía parcial como exposición;

* Prueba de U de Mann-Whitney. 
Tabla 3. Modelo de regresión logística para evaluar factores relacionados a la aparición de lesión de nervio safeno en safenectomías totales. Hospital Militar Central, 2017.

\begin{tabular}{llcc}
\multicolumn{1}{c}{ Características } & Razón de Prevalencias (RP) & $\begin{array}{c}\text { Intervalo de confianza } \\
\text { (IC95\%) }\end{array}$ & $\begin{array}{c}\text { Valor } \boldsymbol{p} \\
\text { Sexo femenino }\end{array}$ \\
$\begin{array}{lll}\text { Edad mayor o igual a } 60 \text { años } \\
\text { Diabetes tipo 2 }\end{array}$ & 0,91 & $0,29-2-82$ & 0,875 \\
Hipertensión arterial & 1,42 & $0,42-4-72$ & 0,563 \\
Obesidad & 5,31 & $0,43-65,10$ & 0,191 \\
Tiempo de enfermedad mayor o igual a & 0,74 & $0,17-3,19$ & 0,695 \\
3 años & 1,23 & $0,22-6,87$ & 0,813 \\
Tiempo quirúrgico menor o igual a 60 & 0,85 & $0,24-2,97$ & 0,805 \\
minutos & & $0,08-0,79$ & 0,018 \\
\hline
\end{tabular}

Razón de prevalencias, tomando la aparición de lesión de nervio safeno como outcome.

proporcional a la edad, siendo la mediana encontrada en nuestro estudio, consistente con otros reportes en Rusia ${ }^{[17]} \mathrm{y}$ Reino Unido ${ }^{[18]}$.

Por otro lado, la mayoría de estudios que han reportado la distribución por género de esta enfermedad, dan resultados contradictorios ${ }^{[4]}$. Sin embargo, se reconoce una discreta asociación con el sexo femenino a nivel mundial ${ }^{[19]}$. Por ejemplo, Edouard et al. ${ }^{[20]}$, en Cuba encontró incidencias del 10 al 20\% para el sexo masculino y del 20 al $50 \%$ para el sexo femenino, mientras que Zolotukhin et al. ${ }^{[17]}$, en Rusia, determinó una frecuencia de $63 \%$ en mujeres. Esta discordancia con respecto a los resultados encontrados se podría explicar por el hecho de que solo se analizó al subgrupo de pacientes que llega a indicación quirúrgica, asociado a que la población que se atiende en el Hospital Militar Central es predominantemente masculina.

En el estudio de posibles comorbilidades asociadas, no se encontró ninguna relación con diabetes tipo 2 o hipertensión arterial, cuya relación con la insuficiencia venosa aún se encuentra en discusión ${ }^{[21,22]}$; ni con obesidad cuyo papel como factor de riesgo ya está reconocido por múltiples estudios a nivel global ${ }^{[2,4,8]}$. Los pacientes con obesidad representaron en nuestro estudio el $16,8 \%$ del total, siendo mayor la proporción en el grupo que recibió safenectomía parcial. Al respecto, no se ha encontrado información sobre la posible influencia de este factor en la eficacia de ambas técnicas; sin embargo, un estudio de seguimiento reciente ha encontrado que la obesidad es un factor de riesgo de recurrencia pos-safenectomia ${ }^{[23]}$, por lo que futuros estudios deben explorar la posibilidad de realizar seguimientos por un mayor lapso de tiempo con pacientes posoperados.

Murad et al. ${ }^{[8]}$, elaboraron un meta-análisis donde se encontró que las complicaciones posoperatorias más frecuentes después del tratamiento quirúrgico fueron hematomas (31\%), lesión del nervio safeno o sural (10-23\%), flebitis superficial (0-12\%) e infección del sitio operatorio (3-6\%). Estos datos difirieron con lo hallado en esta investigación donde la aparición de hematoma varió entre $7-11 \%$ y la infección de sitio operatorio se encontró solamente en un caso ( $1,6 \%$ del total de pacientes). Si bien esto último difiere de lo reportado por el estudio de Murad et al., si es consistente con lo presentado por Decinti-Weiss ${ }^{[24]}$, donde se informa de una estimación de incidencia de infección en heridas operatorias limpias de aproximadamente $2 \%$.

La presencia de hematomas no está necesariamente relacionada al tipo de safenectomía sino a lo extensa de la varicectomía complementaria, al diámetro de la vena safena mayor extirpada, 0 a las venas perforantes insuficientes existentes ${ }^{[10]}$; todas estas variables no fueron incluidas en nuestro estudio, lo que no permite hacer mayores inferencias sobre los resultados obtenidos. Se sugiere que futuros estudios incluyan estas variables y exploren los factores asociados a esta complicación que está presente en un buen porcentaje de pacientes posoperados en nuestra población.

Se encontró diferencias significativas entre los días de hospitalización posoperatoria, siendo este tiempo mayor en el grupo de safenectomía total. Esto se puede relacionar con la mayor presencia de complicaciones, como la lesión del nervio safeno, en este grupo. Así mismo, aunque no se produzca lesión nerviosa durante la cirugía, la manipulación de la vena safena en los dos tercios distales de la pierna provoca la inflamación del lecho quirúrgico que involucra al nervio safeno por su estrecha contigüidad y por ende produce mayor dolor ${ }^{[10]}$. Agregándose a lo mencionado anteriormente, el tiempo de hospitalización postoperatoria se suele considerar como un indicador indirecto de la necesidad de analgesia endovenosa ${ }^{[25]}$; por ello, se puede inferir que el grupo de safenectomía parcial requirió menos analgesia endovenosa en el postoperatorio y por tanto pudieron ser dados de alta con analgésicos orales con mayor prontitud.

De los 64 pacientes a los que se les realizó safenectomía total 31 de ellos $(48,4 \%)$ presentaron sintomatología compatible 
con lesión del nervio safeno, mientras que en los operados por safenectomía parcial fue solamente 1 (2,3\%). Lovecek et al. ${ }^{[26]}$, encontró frecuencias de lesión de nervio safeno para safenectomía parcial y total en $1,6 \%$ y $7 \%$ respectivamente; por otro lado, Morrison et al. reportan frecuencias de hasta $40 \%$ en safenectomias totales ${ }^{[12]}$. En este estudio se mantiene la tendencia de encontrar una mayor frecuencia de lesión de nervio safeno en safenectomía total con un elevado contraste con lo encontrado en el grupo de safenectomia parcial. Esto podría ser explicado por la técnica utilizada por los cirujanos en este servicio.

La extracción de la vena safena en el muslo es segura, es decir no existe relación anatómica entre la vena y el nervio, infragenicularmente el nervio y la vena confluyen progresivamente hasta entrar en contacto en los dos tercios distales de la pierna, esto se basa en la posición anatómica de la vena safena que va acompañado del nervio safeno hasta los dos tercios distales de la pierna ${ }^{[10,12]}$. Sin embargo, cuando la extracción se realizada en el muslo, no existe peligro de lesión nerviosa; a excepción de los casos donde la extracción sobrepasa la articulación de la rodilla en dirección distal ${ }^{[26]}$. Futuras investigaciones deben abarcar la evaluación de la técnica operatoria en detalle y analizar si tiene alguna relación con la aparición de complicaciones posoperatorias.

La mejoría clínica estuvo presente en más del $90 \%$ de pacientes en ambos grupos, no encontrándose diferencia significativa entre grupos. No se encontraron estudios que comparen mejoría clínica entre ambas técnicas quirúrgicas; sin embargo, esta investigación muestra que, de manera indistinta entre una u otra técnica, la gran mayoría de pacientes saldrán de alta satisfechos con los resultados, al menos dentro de los 10 primeros días posoperatorios.

Cabe destacar que este estudio tiene numerosas limitaciones, que en general residen en la inclusión de sesgos de información, debido a que la fuente usada (historias clínicas) a veces no constituye un instrumento de calidad suficiente como para asegurar la confiabilidad de los datos obtenidos. Así mismo, al ser un estudio realizado en un solo establecimiento de salud, los datos no son lo suficientemente representativos para permitir extrapolar conclusiones más allá del ámbito local.

Por otro lado, la operacionalización de la variable "mejoría clínica" se ha basado en la información usualmente disponible en las historias clínicas, debido a que este ha sido un estudio retrospectivo, no se han podido obtener otros índices más confiables como el Venous Clinical Severity Score o una evaluación de calidad de vida mediante cuestionarios validados como VEINES-QOL/Sym ${ }^{[8]}$. Futuros estudios deberán evaluar el uso de diseños prospectivos en donde se pueda evaluar el outcome de mejoría clínica de forma adecuada.

Finalmente, se concluye que los pacientes con safenectomía parcial presentaron mejores desenlaces posoperatorios (frecuencia de complicaciones posoperatorias, lesión del nervio safeno y días de hospitalización) en comparación con los que recibieron safenectomia total en el Hospital Militar Central "Coronel Luis Arias Scheiber", durante el periodo de enero a diciembre del 2017.

Financiamiento: El presente estudio fue autofinanciado.

Conflicto de intereses: Los autores declaran no tener ningún conflicto de intereses.

\section{REFERENCIAS BIBLIOGRÁFICAS}

1. Kasperczak J, Ropacka-Lesiak M, Breborowicz HG. [Definition, classification and diagnosis of chronic venous insufficiency - part II]. Ginekol Pol. 2013;84(1):51-5. [Article in Polish]

2. Eberhardt RT, Raffetto JD. Chronic Venous Insufficiency. Circulation. 2014;130(4):333-46.

3. Eklöf B, Rutherford RB, Bergan JJ, Carpentier PH, Gloviczki P, Kistner $\mathrm{RL}$, et al. Revision of the CEAP classification for chronic venous disorders: consensus statement. J Vasc Surg. 2004;40(6):1248-52.

4. Bergan JJ, Schmid-Schönbein GW, Smith PD, Nicolaides AN, Boisseau MR, Eklof B. Chronic venous disease. N Engl J Med. 2006;355(5):488-98.

5. Navas Hergueta ML, López Laguna AN. Insuficiencia venosa crónica. Actual En Med Fam. 2011;7(9):509-14.

6. Spiridon $M$, Corduneanu $D$. Chronic venous insufficiency: a frequently underdiagnosed and undertreated pathology. Maedica (Buchar). 2017;12(1):59-61.

7. Bozkurt K, Rabe E, Sharkawy MI. Insuficiencia venosa crónica: manejo y tratamiento. EMJ Dermatol. 2017;5(Suppl 3):2-14.

8. Gloviczki P, Comerota AJ, Dalsing MC, Eklof BG, Gillespie DL, Gloviczki ML, et al. The care of patients with varicose veins and associated chronic venous diseases: clinical practice guidelines of the Society for Vascular Surgery and the American Venous Forum. J Vasc Surg. 2011;53(5 Suppl):2S-48S.

9. Chávez-Reyes G, Chirinos-Caraza SJ. Safenectomía ambulatoria segmentaria. Acta Méd Peru. 2006;23(2):56-8.

10. Jaworucka-Kaczorowska A, Oszkinis G, Huber J, Wiertel-Krawczuk A, Gabor E, Kaczorowski P. Saphenous vein stripping surgical technique and frequency of saphenous nerve injury. Phlebology. 2015;30(3):210-6.

11. Hamdan A. Management of varicose veins and venous insufficiency. JAMA. 2012;308(24):2612-21.

12. Morrison C, Dalsing MC. Signs and symptoms of saphenous nerve injury after greater saphenous vein stripping: prevalence, severity, and relevance for modern practice. J Vasc Surg. 2003;38(5):886-90.

13. Ministerio del Trabajo y Promoción del Empleo (MTPE). Boletín estadístico mensual de Notificaciones de Accidentes de Trabajo, Incidentes peligrosos y Enfermedades Ocupacionales. [Internet]. Lima: MTPE; 2013. Disponible en: https://issuu.com/mtpe/docs/ sat_marzo 2013

14. Loli A. Ambiente laboral y condiciones de salud de las enfermeras en los Hospitales de las Fuerzas Armadas, EsSALUD y clínicas particulares de Lima Metropolitana - 1996. An Fac Med. 2000;61(2):163-41.

15. Vargas N, Chávez P, Zamudio S. Guía de Práctica Clínica para Diagnóstico y Tratamiento de la Insuficiencia Venosa Crónica del Hospital Militar Central Coronel Luis Arias Schreiber. Lima: Hospital Militar Central; 2017. 
16. Milone M, Di Minno MN, Maietta P, Shatalova O, Musella M, Milone F. Great saphenous vein stripping and nerve injury: the role of stripping direction. Int Angiol. 2015;34(3):238-42.

17. Zolotukhin IA, Seliverstov EI, Shevtsov YN, Avakiants IP, Nikishkov AS, Tatarintsev AM, et al. Prevalence and risk factors for chronic venous disease in the general Russian population. Eur J Vasc Endovasc Surg. 2017;54(6):752-8

18. Evans CJ, Fowkes FG, Ruckley CV, Lee AJ. Prevalence of varicose veins and chronic venous insufficiency in men and women in the general population: Edinburgh Vein Study. J Epidemiol Community Health. 1999;53(3):149-53.

19. Rabe E, Guex JJ, Puskas A, Scuderi A, Fernandez Quesada F, VCP Coordinators. Epidemiology of chronic venous disorders in geographically diverse populations: results from the Vein Consult Program. Int Angiol. 2012;31(2):105-15.

20. Edouard Otrante $D$, Quiñones Castro M, Borrás Migues M, Rodríguez Villalonga L, Chirino Díaz L. Insuficiencia venosa crónica y calidad de vida. Rev Cuba Angiol Cir Vasc. 2010;11(1):27-33.
21. Rabe E, Berboth G, Pannier F. [Epidemiology of chronic venous diseases]. Wien Med Wochenschr. 2016;166(9-10):260-3. [Article in German]

22. Matic $P$, Jolic $S$, Tanaskovic $S$, Soldatovic I, Katsiki N, Isenovic E, et al. Chronic Venous Disease and Comorbidities. Angiology. 2015;66(6):539-44.

23. Hartmann K, Klode J, Pfister R, Toussaint M, Weingart I, Waldermann $\mathrm{F}$, et al. Recurrent varicose veins: sonography-based re-examination of 210 patients 14 years after ligation and saphenous vein stripping. Vasa. 2006;35(1):21-6.

24. Decinti-Weiss E. Heridas. En: Bases de la medicina clínica. Santiago: Facultad de Medicina. Universidad de Chile; 2010. p. 1-17.

25. Akagi D, Arita H, Komiyama T, Ishii S, Shigematsu K, Nagawa H, et al. Objective assessment of nerve injury after greater saphenous vein stripping. Eur J Vasc Endovasc Surg. 2007;33(5):625-30.

26. Herman J, Lovecek M, Svach I, Duda M. Limited versus total stripping of vena saphena magna. Bratisl Lek Listy. 2002;103(11):434-6.

\section{Las ediciones anteriores de Acta Médica Peruana están disponibles en:}

www.scielo.org.pe

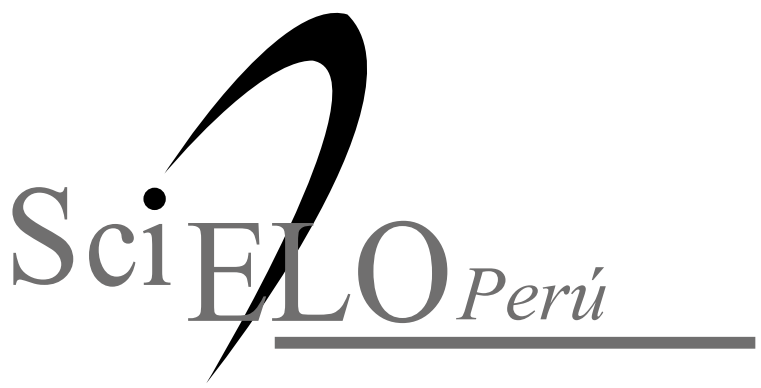

